\title{
Prevalence of malnutrition in paediatric patients admitted to a UK spinal injury centre - an 8-month pilot study
}

\author{
S. S. Wong ${ }^{1,2}$, A. Graham ${ }^{1}$, G. Grimble ${ }^{2}$ and A. Forbes ${ }^{2}$ \\ ${ }^{1}$ National Spinal Injuries Centre, Stoke Mandeville Hospital, Aylesbury, Bucks HP21 8AL, UK and ${ }^{2}$ Centre for \\ Gastroenterology and Clinical Nutrition, University College London, UK
}

\begin{abstract}
Childhood malnutrition affects growth and development, increases susceptibility to nutrition-related complications and increases healthcare costs ${ }^{(1)}$. Different methods have been used to assess nutritional status in children admitted to hospital and there is no agreement as to which is best ${ }^{(2-5)}$. Current data on prevalence of malnutrition (undernutrition and overnutrition) in children with spinal cord injuries (SCI) are limited. The aim of this study was to determine the prevalence of malnutrition in paediatric/adolescent patients with SCI. Undernutrition was defined from the weight for height criteria recommended by Waterlow ${ }^{(5)}$ (normal: $>90 \%$; mild undernutrition: 81-90\%; moderate undernutrition: $70-80 \%$; severe undernutrition: $<70 \%$ ). Overnutrition was defined by using BMI centile chart (overweight: $>91$ st centile, obese: $>98$ th centile). After obtaining ethics approval, data for weight and height were sought in fifty-three children with SCI (mean age: 11.1 years (SD): 4.8; median: 12.0 years, range: $1.0-17.9$ years, $41.5 \%$ female) consecutively admitted to the National Spinal Injuries Centre, Stoke Mandeville Hospital, UK from January to August 2010. On admission, weight for height was obtained in forty children [measured weight $(47,88.6 \%)$ and estimated height $(40,75.5 \%)$ ]. The most common causes of SCI were non-traumatic $(21,52.5 \%)$ followed by road traffic accidents $(16,40.0 \%)$, sports injuries $(2,5 \%)$, falls $(1,2.5 \%)$ and assault $(1,2.5 \%)$. Eight $(20 \%)$ of these children were at risk of undernutrition (weight for height $<90 \%$ ), two $(5 \%)$ were at moderate risk of undernutrition (weight for height $80-90 \%$ ) and two $(5 \%)$ were at high risk of undernutrition (weight for height $<70 \%)$. Nineteen $(47.5 \%)$ were overweight and eleven $(27.5 \%)$ were obese. Only one in three $(33.3 \%)$ of the patients at risk $(26.3 \%$ : overnutrition; $50 \%$ : undernutrition) were referred for nutritional intervention. A very high prevalence of malnutrition was found in this group of patients, suggesting that children with neuro-disabilities (SCI) are vulnerable to malnutrition. Given the adverse consequences for short- and long-term health and well-being a valid and practical tool for nutritional screening is needed in this high risk group. Closer scrutiny of the existing but unvalidated paediatric nutrition-screening tool is therefore being conducted with a larger sample size.
\end{abstract}

The authors would like to thank the patients and ward staff on St. Francis ward, Ebba Bergstrom and Kirsten Hart for height estimation, Wendy Nicholls from Dietetic Department and Pauline Bateman from Medical Records and the Waterloo Foundation and Abbott Nutrition for the financial support. UCL Staff receive support from the CBRC funding awarded to UCL and its partner Trust by NIHR.

1. Brotherton A, Simmonds N \& Stroud M (2010) Malnutrition Matters: Meeting Quality Standards in Nutrition Care. BAPEN, Redditch.

2. McCarthy H, McNutly H, Dixon M et al. (2008) Screening for nutrition risk in children: The validation of a new tool. J Hum Nutr Diet 21, 395-396.

3. Gerasimidis K, Keane O, Macleod I et al. (2010) A four-stage evaluation of the Paediatric Yorkhill Malnutrition Score in a tertiary paediatric hospital and a district general hospital. Br J Nutr 104, 751-756.

4. Joosten K \& Meyer R (2010) Nutritional screening and guidelines for managing the child with faltering growth. Eur J Clin Nutr 64, S22-S24.

5. Waterlow JC (1972) Classification and definition of protein-calorie malnutrition. BMJ 3, 566-569. 